\title{
Intervenção Coronária Percutânea em Octogenários: Houve Mudanças no Perfil dos Casos Tratados nos Últimos Cinco Anos?
}

\author{
Sergio Costa Tavares Filho', Luiz Fernando L. Tanajura', Fausto Feres' ${ }^{1}$, Marinella P. Centemero', \\ Áurea J. Chaves', Andréa S. Abizaid'1', Vinícius Esteves', Gustavo Gama', \\ Amanda G. M. R. Sousa', J. Eduardo Sousa'
}

\section{RESUMO}

Introdução: Com o aumento da longevidade da população, as intervenções coronárias percutâneas (ICP) em octogenários têm sido realizadas com frequência crescente. No entanto, como os pacientes vivem mais, possivelmente apresentar-se-ão ao cardiologista com doença mais avançada, tanto em relação às comorbidades como à própria coronariopatia, fato que, se confirmado, poderia influenciar os resultados almejados. Método: Estudo de coorte, retrospectivo, com o objetivo de avaliar se houve mudanças nos perfis clínico e angiográfico de octogenários tratados por meio de ICP numa fase mais atual (biênio 2006/ 2007), em comparação com o período 2002/2003. Os pacientes foram identificados a partir de um banco de dados informatizado, no qual eram incluídos de forma prospectiva e divididos em dois grupos: grupo A, 128 pacientes tratados no biênio 2006/2007; e grupo B, 98 pacientes dilatados no biênio 2002/2003. Não foram estabelecidos critérios de inclusão e exclusão. Consideraram-se significantes valores de $\mathrm{P}<0,05$. Resultados: Os 128 pacientes do grupo A corresponderam a $4,7 \%$ das ICP realizadas em 2006/2007, enquanto os 98 casos do grupo B corresponderam a $3,7 \%$ das dilatações de 2002/ $2003(P=0,07)$. Predominaram significativamente no grupo A: ICP em pacientes renais crônicos $(28,1 \%$ vs. $10,2 \%$; $\mathrm{P}=0,008)$ e uso de stents com diâmetro $<2,5 \mathrm{~mm}(27,7 \%$ vs. $8,2 \% ; P=0,0001)$. Notamos tendência para tratar maior número de pacientes com doença multiarterial $(60 \%$ vs. $49 \% ; P=0,12)$ e lesões reestenóticas $(6,7 \%$ vs. $1 \%$; $\mathrm{P}=0,09)$. O sucesso do procedimento foi similar entre os grupos $(93,8 \%$ vs. $94,9 \%$, nos grupos A e B, respectivamente; $P>0,99)$. Conclusões: 1) Houve tendência do aumento de octogenários tratados mais recentemente; e 2) os casos dilatados no biênio 2006/2007 apresentaram perfil clínico/angiográfico de maior risco, em especial para o desenvolvimento de reestenose coronariana.

DESCRITORES: Doença das coronárias. Angioplastia transluminal percutânea coronária. Idoso de 80 anos ou mais. Idoso.

\begin{abstract}
Percutaneous Coronary Intervention in EightyYear-Old Individuals: Have There Been Changes in the Profile of Cases Treated in the Last Five Years?

Introduction: As population longevity increases, percutaneous coronary interventions $(\mathrm{PCl})$ in eighty-year-old individuals have been increasingly more performed. However, as patients live longer, they are likely to be referred to the cardiologist with a more advanced disease, both in terms of comorbidities and coronary disease, which might influence the desired results. Method: Retrospective cohort study aimed at assessing whether there have been changes in the clinical and angiographic profile of eighty-year-old individuals treated by PCl at a more recent period (2006/2007) compared to the $2002 / 2003$ period. Patients were prospectively identified and included using a computerized database and were divided into two groups: group A, 128 patients treated in 2006/2007; and group B, 98 patients treated in 2002/2003. There were no inclusion and exclusion criteria. $\mathrm{P}<0.05$ was considered statistically significant. Results: The 128 patients in group A corresponded to $4.7 \%$ of $\mathrm{PCl}$ performed in 2006/2007, whereas the 98 cases in group B corresponded to $3.7 \%$ of $\mathrm{PCl}$ in $2002 / 2003(\mathrm{P}=0.07)$. In group $\mathrm{A}, \mathrm{PCl}$ in chronic renal patients $(28.1 \%$ vs. $10.2 \% ; \mathrm{P}=0.008)$ and the use of stents $<2.5 \mathrm{~mm}(27.7 \%$ vs. $8.2 \%$; $\mathrm{P}=0.0001)$ were statistically significant. There was a trend to treat a larger number of patients with multiartery disease $(60 \%$ vs. $49 \%$; $P=0.12)$ and restenotic lesions $(6.7 \%$ vs. $1 \% ; P=0.09)$. The procedure success rate was similar between groups (93.8\% vs. 94.9\%, respectively; $P>0.99)$. Conclusions: 1 ) There was a trend in the increase of eighty-year-old individuals treated more recently; 2) cases dilated in 2006/2007 had a higher clinical/angiographic risk profile, specially for the development of coronary restenosis.
\end{abstract}

DESCRIPTORS: Coronary disease. Angioplasty, transluminal, percutaneous coronary. Aged, 80 and over. Aged.

\footnotetext{
1 Seção Médica de Angioplastia Coronária, Instituto Dante Pazzanese de Cardiologia - São Paulo, SP, Brasil.

Correspondência: Sergio Costa Tavares Filho. Rua Joaquim Távora, 550/193A - Vila Mariana - São Paulo, SP, Brasil - CEP 04015-011 E-mail: sergiotavares@infonet.com.br

Recebido em: 5/11/2008 • Aceito em: 15/2/2009
} 
A população brasileira, seguindo uma tendência universal, vive o processo de aumento da expectativa de vida. Na última pesquisa realizada pelo Instituto Brasileiro de Geografia e Estatística, em 2003, a média de idade dos brasileiros chegou a 71,3 anos, representando crescimento de 0,8 ano em relação a 2000. Em consequência, observou-se aumento substancial da procura de serviços médicos por parte dessa população, em especial nos casos de maior faixa etária, como os octogenários, caracterizados por doença arterial coronariana mais avançada e maior número de comorbidades ${ }^{1-4}$.

Paralelamente, houve evolução tecnológica dos materiais utilizados na cardiologia intervencionista, possibilitando seu uso com maior segurança e efetividade em pacientes com coronariopatia mais complexa. Dessa forma, pressupõe-se que possa ter havido mudanças expressivas no afluxo e nas características de base de octogenários triados para intervenção coronária percutânea (ICP) no momento atual, comparativamente a épocas passadas ${ }^{5-7}$.

Esta investigação visou ao esclarecimento das eventuais mudanças no perfil clínico/angiográfico de octogenários tratados por meio de ICP no biênio 2006/ 2007, em comparação com o período 2002/2003.

\section{MÉTODO}

Analisamos retrospectivamente todos os pacientes octogenários dilatados em nosso Serviço, nos biênios 2006/2007 (grupo A: 128 pacientes, correspondendo a $4,7 \%$ dos 2.723 pacientes tratados nesse período), cujas características clínicas e angiográficas de base, bem como os dados relacionados ao procedimento, foram comparados aos de pacientes de mesma faixa etária tratados no período 2002/2003 (grupo B: 98 casos, correspondendo a 3,7\% dos 2.648 pacientes dilatados no biênio em questão). Todos foram identificados a partir de um banco de dados informatizado, no qual foram incluídos de forma prospectiva. A natureza do estudo foi observacional.

As ICPs com implante de endopróteses coronarianas foram realizadas pela técnica convencional de liberação ótima, com implante direto ou pré-dilatação, a critério do cardiologista intervencionista.

O esquema farmacológico adjunto consistiu de: 1) heparina não-fracionada, na dose de $100 \mathrm{UI} / \mathrm{kg}$ de peso, administrada imediatamente antes do procedimento com o objetivo de atingir tempo de coagulação ativada de 250 segundos ou mais; 2) ácido acetilsalicílico, com dose de ataque de 500 mg na véspera da intervenção, seguida de $100 \mathrm{mg} / \mathrm{dia}$, indefinidamente; e 3) clopidogrel (dose de ataque na véspera de $300 \mathrm{mg}$, seguida por $75 \mathrm{mg} / \mathrm{dia}$ ) ou ticlopidina (na dose de $500 \mathrm{mg} /$ dia, iniciada três dias antes do procedimento). Nosso Serviço estipulou o emprego do esquema antiplaquetário duplo por pelo menos um mês para os pacientes submetidos a implante de stents não-farmacológicos e, a partir de 2006, por 12 meses para os que receberam modelos com liberação de medicamentos. Anteriormente, os tienopiridínicos eram mantidos por três meses para os stents liberadores de sirolimus e por seis meses para os stents liberadores de paclitaxel. Inibidores da glicoproteína IIb/IIla foram utilizados nas doses habituais, sendo sua indicação deixada a critério do cardiologista intervencionista.

Em relação à análise da angiografia coronariana pré-procedimento, foram consideradas passíveis de tratamento lesões com diâmetro de estenose superior a $50 \%$, pelo critério visual, sempre em presença de isquemia miocárdica, clínica ou detectada por testes funcionais. As lesões-alvo foram classificadas de acordo com American College of Cardiology/American Heart Association Task Force on Practice Guidelines ${ }^{8}$. A função ventricular esquerda foi avaliada pela análise da fração de ejeção global do ventrículo esquerdo. A angiografia coronariana quantitativa não foi realizada de forma rotineira, o mesmo acontecendo com o ultrassom intracoronariano.

As ICPs foram realizadas por meio de implante ótimo dos stents coronarianos, com ou sem pré-dilatação. A via de acesso, o modelo de endoprótese a ser implantado e o número de vasos a serem tratados ficaram a critério do cardiologista intervencionista que realizou o procedimento. A hemostasia foi sempre realizada de forma manual.

\section{Definições}

Considerou-se sucesso primário a obtenção de estenose residual inferior a $10 \%$ pelo critério visual, na ausência de complicações maiores (óbito, infarto do miocárdio ou cirurgia de revascularização em caráter de urgência) na fase hospitalar. Infarto do miocárdio foi caracterizado pela presença de novas ondas Q no eletrocardiograma de 12 derivações e/ou por elevações da creatina quinase fração MB (CK$\mathrm{MB})$ superiores a três vezes o valor normal. Insuficiência renal crônica foi definida como clearance da creatinina $<60 \mathrm{ml} / \mathrm{min} / 1,73 \mathrm{~m}^{2}$. Doença coronariana multiarterial foi definida pela presença de lesões coronarianas significativas (> 50\%) em pelo menos dois dos vasos principais e/ou em seus ramos. E ICP estagiada caracterizou-se por intervenções para o tratamento de múltiplos vasos realizadas em procedimentos distintos.

\section{Análise estatística}

As variáveis quantitativas foram expressas por média e desvio padrão e comparadas com o teste $t$ de Student. As variáveis qualitativas foram expressas por porcentuais e comparadas com o teste de qui-quadrado. Consideraram-se significantes valores de $\mathrm{P}<0,05$, sendo todos os testes bicaudais. Para a análise estatística foi utilizado o programa SPSS 13.0. 


\section{RESULTADOS}

Observamos tendência de crescimento na proporção de octogenários tratados no biênio 2006/2007 $(4,7 \%$ vs. $3,7 \% ; P=0,07)$.

Em relação às características clínicas de base (Tabela 1), a maioria igualmente distribuída entre os grupos, notamos que cerca de $40 \%$ dos pacientes eram do sexo feminino, com alta prevalência de fatores de risco para doença arterial coronariana. Pelo menos $75 \%$ dos pacientes eram portadores de hipertensão arterial, um quarto deles era de diabéticos, e quase metade era de tabagistas. A insuficiência renal crônica foi mais comum no grupo A ( $28,1 \%$ vs. $10,2 \%$; $P=0,008)$. A condição clínica mais frequentemente tratada foi angina estável/isquemia silenciosa $(68,8 \%$ vs. $60,3 \% ; \mathrm{P}=0,44)$.

Quanto ao perfil angiográfico (Tabela 2), observamos tendência a tratar maior número de pacientes com doença multiarterial e lesões reestenóticas nos casos mais recentes. Também nos chamou a atenção o fato de que quase metade dos pacientes apresentava algum grau de disfunção ventricular esquerda $(46,9 \%$ vs. $44,9 \% ; P=0,21)$.

Todas as ICPs foram realizadas pela via femoral. Múltiplos vasos foram tratados em cerca de $20 \%$ dos pacientes (Figura 1), tendo uma minoria deles (Figura 2) recebido stents farmacológicos $(10,7 \%$ vs. $13,2 \%$; $\mathrm{P}=0,47)$. Stents de menor calibre $(<2,5 \mathrm{~mm})$ foram mais frequentemente utilizados no último biênio $(27,6 \%$ vs. $8,2 \% ; P=0,0001$ ) (Figura 3 ).
$\mathrm{Na}$ fase hospitalar, o sucesso do procedimento foi semelhante entre os grupos $(93,8 \%$ vs. $94,9 \%$ nos grupos A e B, respectivamente; $P>0,99$ ). Houve um óbito hospitalar no grupo A, decorrente de morte súbita. Não ocorreram casos de infarto ou cirurgia de revascularização de emergência.

\section{DISCUSSÃO}

Esta investigação demonstrou aumento de 27\% de octogenários tratados por ICP nos últimos anos, comparativamente ao início da década, apesar de tais casos ainda serem pouco comuns, não atingindo 5\% do número total de pacientes.

Os octogenários atualmente dilatados são de fato mais complexos, tanto do ponto de vista clínico (mais casos com disfunção renal) como angiográfico, pois exibem maior porcentual de múltiplos vasos tratados e lesões em artérias coronárias de menor calibre. Nossa expectativa de maior utilização dos modelos de liberação de medicamentos em razão desse perfil mais predisposto à reestenose não foi confirmada, possivelmente em razão da limitação imposta pela Previdência Social ao emprego dessas próteses em hospitais públicos.

Até o final da década passada, havia questionamentos quanto à indicação de ICP em idosos, dúvida ainda mais pertinente nos pacientes com idade muito avançada. No entanto, ensaios randomizados realizados na vigência de insuficiência coronariana tanto aguda como crônica ${ }^{9}$ deram respaldo à indicação de ICP nessas populações, justificando o crescimento de casos tratados na atualidade.

TABELA 1

Características clínicas basais dos pacientes tratados

\begin{tabular}{|c|c|c|c|}
\hline & Grupo A & Grupo B & $\mathbf{P}$ \\
\hline Número total de pacientes & 2.723 & 2.648 & \\
\hline Número de octogenários & 128 & 98 & \\
\hline Idade, anos (média \pm desvio padrão) & $82,9 \pm 2,9$ & $82,8 \pm 2,7$ & $>0,99$ \\
\hline Sexo feminino, $\%$ & 39 & 40,8 & 0,89 \\
\hline Hipertensão arterial, \% & 82,8 & 75,5 & 0,23 \\
\hline Diabetes melito, \% & 29,6 & 24,4 & 0,45 \\
\hline Tabagismo, \% & 41,4 & 52 & 0,13 \\
\hline Dislipidemia, \% & 42,18 & 39,8 & 0,72 \\
\hline IAM prévio, \% & 35,9 & 32,6 & 0,67 \\
\hline História familiar de DAC, \% & 25 & 35,6 & 0,10 \\
\hline Insuficiência renal crônica, \% & 28,1 & 10,2 & 0,008 \\
\hline Quadro clínico, \% & & & 0,44 \\
\hline Angina estável/ isquemia silenciosa, \% & 68,8 & 60,3 & \\
\hline Angina instável, \% & 24,2 & 33,6 & \\
\hline Infarto do miocárdio, \% & 7 & 6,1 & \\
\hline
\end{tabular}

$\mathrm{DAC}=$ doença arterial coronariana; IAM = infarto agudo do miocárdio. 
TABELA 2

Perfil angiográfico dos pacientes tratados

\begin{tabular}{lccc}
\hline & Grupo A (128 pacientes/133 vasos) & Grupo B $\mathbf{( 9 8}$ pacientes/105 vasos) & P \\
\hline Doença multiarterial, \% & 60,1 & 48,9 & 0,10 \\
FEVE < 55\%, \% & $46,9 \%$ & $44,9 \%$ & 0,21 \\
Vaso tratado, \% & & & 0,15 \\
DA & 40,6 & 29,5 & \\
CX & 24,1 & 20 & \\
CD & 27,1 & 41,9 & 0,23 \\
Ponte de safena & 8,2 & 8,6 & \\
Tipo de lesão, \% & 69,1 & 73,7 & 0,08 \\
A/B1 & 30,9 & 26,3 & 1 \\
B2/C & 6,7 & & \\
Lesão reestenótica, \% & & \\
CD = artéria coronária direita; CX = artéria circunflexa; DA = artéria descendente anterior; FEVE = fração de ejeção do ventrículo \\
esquerdo.
\end{tabular}

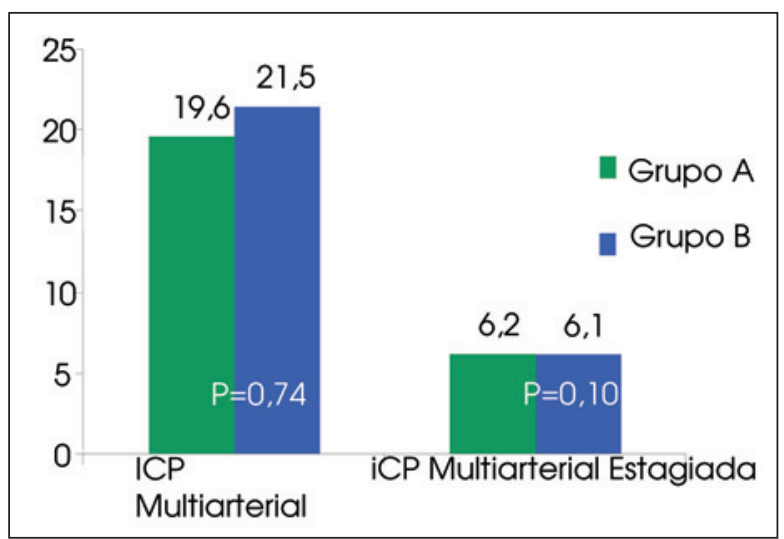

Figura 1 - Proporção de intervenção coronária percutânea (ICP) multiarterial e estagiada da população estudada.

Alguns estudos menores avaliaram o benefício da angioplastia em octogenários ${ }^{10-15}$, mas foi o Trial of Invasive versus Medical Therapy in the Elderly (TIME) ${ }^{16}$, primeiro grande estudo randomizado a comparar revascularização miocárdica (quase dois terços dos procedimentos realizados por meio de ICP) ou tratamento clínico otimizado em octogenários com angina crônica estável, que nos forneceu as primeiras informações consistentes. Nos casos alocados para a estratégia de revascularização miocárdica, observaram-se reduções significantes nos porcentuais de hospitalizações $(18 \%$ vs. $72 \%$; $P<0,001)$, de hospitalizações ocorridas pela necessidade de procedimentos de revascularização $(10 \%$ vs. 48\%; P < 0,01), e de eventos cardíacos maiores $(26 \%$ vs. 64\%; $\mathrm{P}<0,001)$. Análise de um subgrupo de 188 pacientes demonstrou que essas vantagens ocorreram sem aumento das despesas médicas ${ }^{17}$. Por fim, o relato de quatro anos de evolução clínica tardia ratificou a vantagem da estratégia de

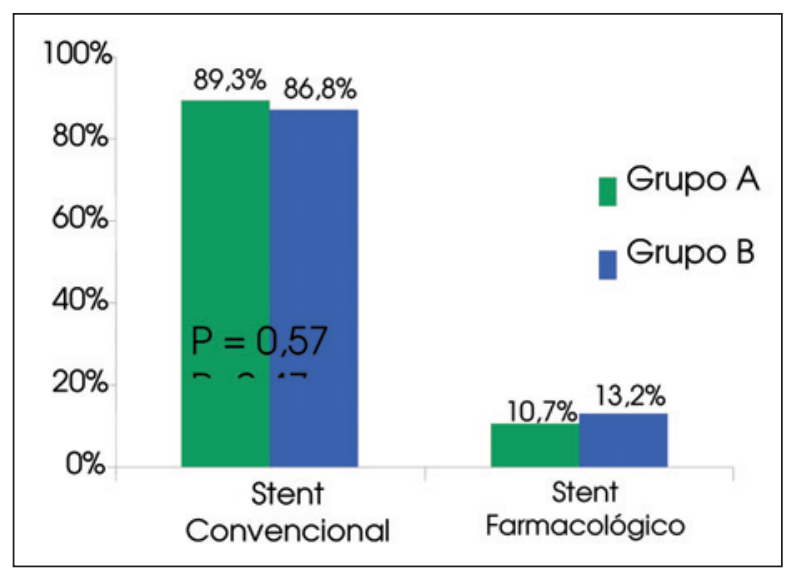

Figura 2 - Proporção de stents convencionais e farmacológicos.

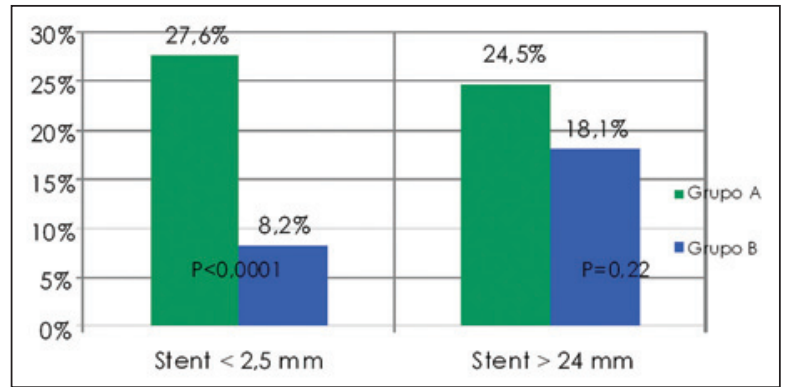

Figura 3 - Calibre dos stents.

revascularizar os octogenários, uma vez que a nãorevascularização no primeiro ano do ensaio foi preditor independente de mortalidade. Os pacientes revascularizados utilizaram menos medicamentos para o controle da angina $(P<0,001)^{18}$. 
Tavares Filho SC, et al. Intervenção Coronária Percutânea em Octogenários: Houve Mudanças no Perfil dos Casos Tratados nos Últimos Cinco Anos? Rev Bras Cardiol Invas. 2009;17(1):76-81.

O registro Alberta Provincial Project for Outcomes Assessment in Coronary Heart Disease (APPROACH) ${ }^{19}$, posteriormente, avaliou o tratamento de 6.181 pacientes idosos portadores de angina estável, $16 \%$ dos quais com idade superior a 80 anos, no tocante à mortalidade. Os octogenários tratados por meio de ICP apresentaram diferença absoluta de mortalidade de 11,3\% em relação aos mantidos apenas com o tratamento clínico, após acompanhamento de quatro anos, números superiores aos observados em faixas etárias menores, sugerindo maior benefício nos mais idosos.

Outros relatos analisaram os octogenários com doença coronariana sob outros prismas. Liistro e Colombo ${ }^{20}$, além de citarem dados similares aos nossos (aumento do número absoluto de casos e da complexidade clínica dos mesmos), aferiram sobrevida livre de eventos em cinco anos após revascularização semeIhante, nos diferentes grupos etários $(78 \%$ para pacientes $<70$ anos vs. $75 \%$ para aqueles $>80$ anos). Também nos pareceu relevante a informação da sobrevida semelhante aos cinco anos de evolução para as diferentes técnicas de revascularização $(81,4 \%$ para ICP vs. $85,7 \%$ para cirurgia), excetuando-se os diabéticos, mais beneficiados com revascularização cirúrgica. Por fim, Lemos et al. ${ }^{21}$ identificaram, em 246 octogenários tratados entre 1998 e 2003, os seguintes preditores de mortalidade: infarto do miocárdio como apresentação inicial da coronariopatia, e doença triarterial na cinecoronariografia diagnóstica.

\section{Limitações}

Este estudo apresenta as seguintes limitações: 1) a análise foi feita de forma retrospectiva, ou seja, tratase de um estudo comparativo entre duas coortes de pacientes, com todas as restrições aplicadas a esse tipo de análise; 2) não foi realizado seguimento tardio dos pacientes; 3) pela limitação ao uso dos stents farmacológicos, as indicações desses modelos, padrão de referência na atualidade, não puderam ser avaliadas de forma precisa, detalhe relevante, em razão de as lesões-alvo tratadas na atualidade serem mais predispostas a reestenose.

\section{CONCLUSÕES}

Seguindo tendência universal, observou-se aumento do número de octogenários tratados mais recentemente por meio de ICP, os quais exibem perfil mais complexo do ponto de vista clínico e angiográfico, sobretudo para o desenvolvimento de reestenose coronariana.

\section{CONFLITO DE INTERESSES}

Os autores declararam inexistência de conflito de interesses.

\section{REFERÊNCIAS BIBLIOGRÁFICAS}

1. Nasri F. O envelhecimento populacional no Brasil. Einstein. 2008;6(Supl. 1):S4-S6.
2. Veras RP, Ramos LR, Kalache A. Crescimento da população idosa no Brasil: transformações e consequências na sociedade. Rev Saúde Pública. 1987;21(3):225-33.

3. Carvalho JAM, Garcia RA. O envelhecimento da população brasileira: um enfoque demográfico. Cad Saúde Pública. 2003;19(3):725-33.

4. Instituto Brasileiro de Geografia e Estatística. Censo 2005. São Paulo: IBGE; 2005.

5. Caixeta A, Abizaid A, Aoki J, Dangas G, Mehran R. Future stent drug delivery systems. Minerva Cardioangiol. 2008;56(1): 155-66.

6. Abizaid A, César FB, Silva EM, Feres F, Mattos LAP, Staico $\mathrm{R}$, et al. Stents farmacológicos: novos fármacos. Rev Bras Cardiol Invas. 2004;12(2):96-101.

7. Costa Jr JR, Carlier SG, Costa R, Sano K, Kimura M, Tanaka $\mathrm{K}$, et al. Novas modalidades de imagem em cardiologia intervencionista: tomografia óptica, angiografia tridimensional e histologia virtual. Rev Bras Cardiol Invas. 2006;14(2):156-62.

8. Antman EM, Anbe DT, Armstrong PW, Bates ER, Green LA, Hand $M$, et al. ACC/AHA guidelines for the management of patients with ST-elevation myocardial infarction - executive summary: a report of the American College of Cardiology/ American Heart Association Task Force on Practice Guidelines (Writing Committee to Revise the 1999 Guidelines for the Management of Patients With Acute Myocardial Infarction). Circulation. 2004;110(5):588-636.

9. Cannon CP, Weintraub WS, Demopoulos LA, Vicari R, Frey M), Lakkis N, et al.; TACTICS (Treat Angina with Aggrastat and Determine Cost of Therapy with an Invasive or Conservative Strategy): Thrombolysis in Myocardial Infarction 18 Investigators. Comparison of early invasive and conservative strategies in patients with unstable coronary syndromes treated with the glycoprotein Ilb/Illa inhibitor tirofiban. $N$ Engl J Med. 2001;344(25):1879-87.

10. Batchelor WB, Anstrom KJ, Muhlbaier LH, Grosswald R, Weintraub WS, O'Neill WW, et al. Contemporary outcome trends in the elderly undergoing percutaneous coronary interventions: results in 7,472 octogenarians. National Cardiovascular Network Collaboration. J Am Coll Cardiol. 2000;36(3): 723-30.

11. Maiello L, Colombo A, Gianrossi R, Thomas J, Finci L. Results of coronary angioplasty in patients aged 75 years and older. Chest. 1992;102(2):375-9.

12. Seto TB, Taira DA, Berezin R, Chauhan MS, Cutlip DE, Ho $\mathrm{KK}$, et al. Percutaneous coronary revascularization in elderly patients: impact on functional status and quality of life. Ann Intern Med. 2000;132:955-8.

13. Palomo Villada JA, Solorio S, Farell Campa J, Abundes Velasco A, Ledesma Velasco M. Immediate results of coronary stent implants in octogenarian patients. Arch Inst Cardiol Mex. 1999;69(5):445-53.

14. Schiano P, Monségu J. Coronary angioplasty in octagenarians. Ann Cardiol Angeiol (Paris). 2008;57(6):365-70.

15. De Servi S, Cavallini C, Dellavalle A, Santoro GM, Bonizzoni E, Marzocchi A, et al.; ROSAI-2 Investigators. Non-ST-elevation acute coronary syndrome in the elderly: treatment strategies and 30-day outcome. Am Heart J. 2004;147(5):830-6.

16. Pfisterer $M$, Buser $P$, Osswald S, Allemann U, Amann W, Angehrn $W$, et al. Trial of invasive versus medical therapy in elderly patients (time) investigators: outcome of elderly patients with chronic symptomatic coronary artery disease with an invasive versus optimized medical treatment strategy: one-year results of the randomized TIME trial. JAMA. 2003;289(9):1117-23.

17. Claude J, Schindler C, Kuster GM, Schwenkglenks M, Szucs $\mathrm{T}$, Buser $\mathrm{P}$, et al. Trial of Invasive versus Medical therapy in 
Tavares Filho SC, et al. Intervenção Coronária Percutânea em Octogenários: Houve Mudanças no Perfil dos Casos Tratados nos Últimos Cinco Anos? Rev Bras Cardiol Invas. 2009;17(1):76-81.

the Elderly (TIME) Investigators. Cost-effectiveness of invasive versus medical management of elderly patients with chronic symptomatic coronary artery disease. Findings of the randomized trial of invasive versus medical therapy in elderly patients with chronic angina (TIME). Eur Heart J. 2004;25(24):2195-203.

18. Pfisterer M. Trial of Invasive versus Medical therapy in Elderly patients Investigators. Long-term outcome in elderly patients with chronic angina managed invasively versus by optimized medical therapy: four-year follow-up of the randomized Trial of Invasive versus Medical therapy in Elderly patients (TIME). Circulation. 2004;110(10):1213-8.
19. Graham MM, Ghali WA, Faris PD, Galbraith PD, Norris CM, Knudtson ML; Alberta Provincial Project for Outcomes Assessment in Coronary Heart Disease (APPROACH) Investigators. Survival after coronary revascularization in the elderly. Circulation. 2002;105(20):2378-84.

20. Liistro F, Colombo A. Coronary angioplasty in elderly patients. Ital Heart J Suppl. 2002;3(1):1-8.

21. Lemos PA, Campos CAH, Ribeiro EE, Falcão LAA, Perin MA, Kajita LJ, et al. Incidência e preditores de óbito precoce e tardio em octogenários brasileiros tratados com intervenção coronária percutânea. Rev Bras Cardiol Invas. 2007;15(1):31-4. 